\title{
A NOTE ON SINGULAR INTEGRALS WITH WEIGHTS
}

\author{
DOUGLAS S. KURTZ AND RICHARD L. WHEEDEN ${ }^{1}$
}

\begin{abstract}
We prove two results concerning the behavior on weighted $L^{p}$ spaces of Calderón-Zygmund singular integrals formed with kernels whose $L^{1}$ moduli of continuity satisfy the Dini condition. We also prove a result about the behavior of multiplier operators.
\end{abstract}

Let $K(x), x \in \mathbf{R}^{n}$, be homogeneous of degree $-n$, have integral over $|x|=1$ equal to zero,and satisfy the $L^{1}$-Dini condition

$$
\int_{0}^{1} \frac{\omega(\delta)}{\delta} d \delta<+\infty, \quad \text { where } \omega(\delta)=\sup \int_{\left|x^{\prime}\right|=1}\left|K\left(\rho x^{\prime}\right)-K\left(x^{\prime}\right)\right| d x^{\prime},
$$

the sup being taken over all rotations $\rho$ of the unit sphere with magnitude at most $\delta$. Let $T f(x)=$ p.v. $(f * K)(x)$ denote the corresponding Calderón-Zygmund singular integral. Under the mild restriction (1), very little is known about the classes of nonnegative weight functions $w(x)$ for which either

$$
\left(\int_{\mathbf{R}^{n}}|T f(x)|^{p} w(x) d x\right)^{1 / p}<c\left(\int_{\mathbf{R}^{n}}|f(x)|^{p} w(x) d x\right)^{1 / p}, \quad 1<p<\infty,
$$

or

$$
\int_{\{x:|T f(x)|>\lambda\}} w(x) d x<\frac{c}{\lambda} \int_{\mathbf{R}^{n}}|f(x)| w(x) d x, \quad \lambda>0, p=1,
$$

where $c$ is a constant independent of $f$ and $\lambda$. Most such known results rely on stronger assumptions about $K$; see, e.g., [5]. If $K$ only satisfies (1), both (2) and (3) are of course valid if $w(x) \equiv 1$ (see [1]); in this case the full strength of (1) is used only to obtain (3), (2) being true under the weaker assumption that $K$ is of class $L \log ^{+} L(|x|=1)([1],[2])$. If $w(x)=|x|^{\alpha}$, a result in [7] states that if $K$ is just in $L \log ^{+} L(|x|=1)$, then (2) holds if $-1<\alpha<p-1$, but does not hold if $\alpha>$ $p-1$ or $\alpha<-1$. This range of $\alpha$ is considerably more restricted than the range $-n<\alpha<n(p-1)$ for which $w(x)=|x|^{\alpha}$ satisfies the $A_{p}$ condition

$$
\left(\frac{1}{|Q|} \int_{Q} w(x) d x\right)\left(\frac{1}{|Q|} \int_{Q} w(x)^{-1 /(p-1)} d x\right)^{p-1}<c,
$$

where $Q$ is any cube in $\mathbf{R}^{n}$.

The main purpose of the note is to prove two facts about $T$ when $K$ satisfies (1). These are related to the result in [7] quoted above. We will show that if $p=1$, an

Received by the editors November 19, 1979.

AMS (MOS) subject classifications (1970). Primary 44A25, 42A18.

${ }^{1}$ Research supported in part by NSF grants MCS-7900918 and MCS-7703980. 
analogous weak-type result (3) holds if $K$ satisfies the stronger assumption (1), and that (2) (or (3) when $p=1$ ) fails for $\alpha>p-1$ or $\alpha<-1$ even if $K$ satisfies (1). Specifically, we will prove

THEOREM 1. Let $K(x)$ be homogeneous of degree - $n$, have mean-value zero on the unit sphere, and satisfy (1). If $-1<\alpha<0$, then (3) holds with $w(x)=|x|^{\alpha}$.

THEOREM 2. There is a kernel $K$ which satisfies the assumptions listed in Theorem 1 and for which (2) and (3) fail when $w(x)=|x|^{\alpha}$ with $\alpha>p-1$ or $\alpha<-1$.

We also prove a result of a different nature which is related to a theorem in [5]. There we consider multiplier operators $T$ defined by $\widehat{T f}(x)=m(x) \hat{f}(x)$, where $m$ is a bounded function which satisfies

$$
\sup _{R>0}\left(R^{s|\alpha|-n} \int_{R<|x|<2 R}\left|D^{\alpha} m(x)\right|^{s} d x\right)^{1 / s}<+\infty
$$

for all multi-indices $\alpha=\left(\alpha_{1}, \ldots, \alpha_{n}\right)$ of nonnegative integers with length $|\alpha|=\alpha_{1}$ $+\cdots+\alpha_{n} \leqslant l$, where $l$ is a preassigned positive integer and $s>1$. The collection of such $m$ is denoted $M(s, l)$, and the result in [5] in question states that if $m \in M(s, l), 1<s \leqslant 2,[n / s]<l<n$ and $n / l<p<(n / l)^{\prime}$, then (2) is valid for $w(x)=|x|^{\alpha}$ if $-n<\alpha<n(p-1)$. Since this is precisely the range of powers of $|x|$ which satisfy the $A_{p}$ condition, it seemed plausible that (2) might hold for any $w$ in $A_{p}$. This, however, is not the case.

TheOREM 3. Let $1<s<2,[n / s]<l<n$ and $n / l<p<(n / l)^{\prime}$. Then there exist $w$ in $A_{p}$ and $m$ in $M(s, l)$ such that the multiplier operator defined by $\widehat{T f}=m \hat{f}$ does not satisfy (2).

The example we will give for Theorem 3 arose in discussions with R. Hunt, B. Muckenhoupt and W.-S. Young.

In what follows, we write $f \in L_{w}^{p}$ if the right side of (2) is finite, and use $c$ to denote constants which may be different in different occurrences.

Proof of Theorem 1.

LEMMA 1. Let $\Omega(x)$ be homogeneous of degree zero and integrable on $|x|=1$. If $\alpha>-1$ and $|t|<2|y|$, then

$$
\int_{|x|<10|y|}|\Omega(x)||x+t+y|^{\alpha} d x<c\|\Omega\|_{L^{\prime}(|x|=1)}|y|^{n+\alpha} .
$$

This is a special case of Lemma 1 in [7].

In the next lemma, we use the known fact that (1) implies (in fact, by [3], is equivalent to)

$$
\int_{|x|>2|y|}|K(x-y)-K(x)| d x<c, \quad y \in \mathbf{R}^{n} .
$$

LemMA 2. Let $K$ satisfy (1) and $-1<\alpha<0$. There is a constant $c$ so that if $Q$ is a cube with center $y_{Q}$, then for $y \in Q$

$$
\int_{\left|x-y_{Q}\right|>2 \operatorname{diam} Q}\left|K(x-y)-K\left(x-y_{Q}\right)\right||x|^{\alpha} d x<c \frac{1}{|Q|} \int_{Q}|x|^{\alpha} d x .
$$


Proof. Let $d=\operatorname{diam} Q$. If $d>2\left|y_{Q}\right|$, then

$$
\frac{1}{|Q|} \int_{Q}|x|^{\alpha} d x>\frac{c}{d^{n}} \int_{|x|<d / 4}|x|^{\alpha} d x>c d^{\alpha} .
$$

Since $\left|x-y_{Q}\right|>2 d$ implies $|x| \geqslant\left|x-y_{Q}\right|-\left|y_{Q}\right|>2 d-d / 2>d$, using the fact that $\alpha<0$ together with (5), we get

$$
\begin{aligned}
\int_{\left|x-y_{Q}\right|>2 d} \mid K(x-y)- & \left.K\left(x-y_{Q}\right)|| x\right|^{\alpha} d x \\
& <d^{\alpha} \int_{\left|x-y_{Q}\right|>2\left|y-y_{Q}\right|}\left|K(x-y)-K\left(x-y_{Q}\right)\right| d x \leqslant c d^{\alpha} .
\end{aligned}
$$

If $d<2\left|y_{Q}\right|$, then

$$
\frac{1}{|Q|} \int_{Q}|x|^{\alpha} d x>\frac{c}{d^{n}} \int_{\left|x-y_{Q}\right|<d / 4}|x|^{\alpha} d x>c\left|y_{Q}\right|^{\alpha} .
$$

Also, if $y \in Q$,

$$
\begin{gathered}
\int_{\left|x-y_{Q}\right|>4\left|y_{Q}\right|}\left|K(x-y)-K\left(x-y_{Q}\right)\right||x|^{\alpha} d x<\left|y_{Q}\right|^{\alpha} \\
\cdot \int_{\left|x-y_{Q}\right|>4\left|y_{Q}\right|}\left|K(x-y)-K\left(x-y_{Q}\right)\right| d x<c\left|y_{Q}\right|^{\alpha},
\end{gathered}
$$

by the reasoning above, since $4\left|y_{Q}\right|>2 d>2\left|y-y_{Q}\right|, y \in Q$. Thus, we have only to show that

$$
\int_{2 d<\left|x-y_{Q}\right|<4\left|y_{Q}\right|}\left|K(x-y)-K\left(x-y_{Q}\right)\right||x|^{\alpha} d x<c\left|y_{Q}\right|^{\alpha}, \quad y \in Q,
$$

or, by a change of variables, that

$$
\Phi=\int_{2 d<|x|<4\left|y_{Q}\right|}|K(x-\delta)-K(x)|\left|x+y_{Q}\right|^{\alpha} d x<c\left|y_{Q}\right|^{\alpha}
$$

where $|\delta|<d<2\left|y_{Q}\right|$.

Consider first the case $d / 2<\left|y_{Q}\right|<4 d$. Note that $\left\{x: 2 d<|x+\delta|<4\left|y_{Q}\right|\right\}$ $\subset\left\{x:\left|y_{Q}\right| / 4<|x|<6\left|y_{Q}\right|\right\}$. Thus, by a change of variables, we have

$$
\begin{aligned}
\Phi & <\int_{2 d<|x|<4\left|y_{Q}\right|}|K(x-\delta)|\left|x+y_{Q}\right|^{\alpha} d x+\int_{\left|y_{Q}\right| / 2<|x|<4\left|y_{Q}\right|}|K(x)|\left|x+y_{Q}\right|^{\alpha} d x \\
& <\int_{\left|y_{Q}\right| / 4<|x|<6\left|y_{Q}\right|}|K(x)|\left|x+\delta+y_{Q}\right|^{\alpha} d x+\int_{\left|y_{Q}\right| / 2<|x|<4\left|y_{Q}\right|}|K(x)|\left|x+y_{Q}\right|^{\alpha} d x .
\end{aligned}
$$

By the homogeneity of $K, K(x)=\Omega\left(x^{\prime}\right) /|x|^{n}$ where $x^{\prime}=x /|x|$. Therefore,

$$
\begin{aligned}
\Phi< & \int_{\left|y_{Q}\right| / 4<|x|<6\left|y_{Q}\right|} \frac{\left|\Omega\left(x^{\prime}\right)\right|}{|x|^{n}}\left|x+\delta+y_{Q}\right|^{\alpha} d x \\
& +\int_{\left|y_{Q}\right| / 2<|x|<4\left|y_{Q}\right|} \frac{\left|\Omega\left(x^{\prime}\right)\right|}{|x|^{n}}\left|x+y_{Q}\right|^{\alpha} d x \\
& <c\left|y_{Q}\right|^{\alpha},
\end{aligned}
$$

by factoring out $|x|^{-n}<c\left|y_{Q}\right|^{-n}$ and applying Lemma 1 . 
If $4 d<\left|y_{Q}\right|$, we write

$$
\begin{aligned}
\Phi= & \int_{2 d<|x|<\left|y_{Q}\right| / 2}|K(x-\delta)-K(x)|\left|x+y_{Q}\right|^{\alpha} d x \\
& +\int_{\left|y_{Q}\right| / 2<|x|<4\left|y_{Q}\right|}|K(x-\delta)-K(x)|\left|x+y_{Q}\right|^{\alpha} d x .
\end{aligned}
$$

Using the fact that $\left|x+y_{Q}\right|$ is equivalent to $\left|y_{Q}\right|$ for $|x|<\left|y_{Q}\right| / 2$ and applying (5), we see the first term on the right is bounded by $c\left|y_{Q}\right|^{\alpha}$. We get the same estimate for the second term by repeating the previous argument and using the fact that $|\delta|<\left|y_{Q}\right| / 4$.

To prove Theorem 1, let $f^{*}$ denote the Hardy-Littlewood maximal function of $f$, $f>0$. The procedure in [8, p. 19], allows us to decompose $\left\{x: f^{*}(x)>\lambda\right\}$ into a sequence of nonoverlapping cubes $\left\{Q_{k}\right\}$ such that $f(x)<\lambda$ for a.e. $x$ outside $\cup Q_{k}$ and $\int_{Q_{k}} f(x) d x /\left|Q_{k}\right| \leqslant c \lambda$. The proof of Theorem 1 is now fairly standard and we shall be brief. Using the $Q_{k}$ above, write $f=g+b$ where $g=f$ outside $\cup Q_{k}$ and $g=\int_{Q_{k}} f(x) d x /\left|Q_{k}\right|$ on $Q_{k}$. Applying Theorem 1 of [7] and the fact that $0<g<$ $\lambda$, we have

$$
\int_{\{x:|T g(x)|>\lambda\}}|x|^{\alpha} d x<\frac{c}{\lambda^{2}} \int_{\mathbf{R}^{n}} g(x)^{2}|x|^{\alpha} d x<\frac{c}{\lambda} \int_{\mathbf{R}^{n}} g(x)|x|^{\alpha} d x
$$

Since $-1<\alpha<0,|x|^{\alpha}$ satisfies the $A_{1}$ condition $\int_{Q}|x|^{\alpha} d x /|Q|<c$ ess $Q^{\text {inf }}|x|^{\alpha}$. Hence, by the definition of $g$,

$$
\begin{aligned}
\int_{\mathbf{R}^{n}} g(x)|x|^{\alpha} d x & =\int_{\left(\cup Q_{k}\right)^{\prime}} f(x)|x|^{\alpha} d x+\sum_{k} \frac{1}{\left|Q_{k}\right|} \int_{Q_{k}} f(t) d t \int_{Q_{k}}|x|^{\alpha} d x \\
& \leqslant c \int_{\mathbf{R}^{n}} f(x)|x|^{\alpha} d x .
\end{aligned}
$$

Let $Q_{k}^{*}$ be $Q_{k}$ expanded concentrically twice. Then

$$
\int_{\cup Q_{k}^{*}}|x|^{\alpha} d x \leqslant c \int_{\cup Q_{k}}|x|^{\alpha} d x \leqslant \frac{c}{\lambda} \int_{\mathbf{R}^{n}} f(x)|x|^{\alpha} d x
$$

the last inequality being a corollary of the weak-type result in [6] for the Hardy-Littlewood maximal function.

Finally, let $y_{k}$ be the center of $Q_{k}$. Since $\int_{Q_{k}} b(y) d y=0$,

$$
\begin{aligned}
\int_{\mathbf{R}^{n}-\cup Q_{k}^{*}}|(K * b)(x)||x|^{\alpha} d x & =\int_{\mathbf{R}^{n}-\cup Q_{k}^{*}}\left|\sum \int_{Q_{k}} K(x-y) b(y) d y\right||x|^{\alpha} d x \\
= & \int_{\mathbf{R}^{n}-\cup Q_{k}^{*}}\left|\sum \int_{Q_{k}}\left\{K(x-y)-K\left(x-y_{k}\right)\right\} b(y) d y\right||x|^{\alpha} d x .
\end{aligned}
$$


Changing the order of integration, applying Lemma 2 and the definition of $A_{1}$, we have

$$
\begin{aligned}
\int_{\mathbf{R}^{n}-\cup Q_{k}^{*}}|(K * b)(x)||x|^{\alpha} d x \\
\quad<\sum \int_{Q_{k}}|b(y)|\left(\int_{\mathbf{R}^{n}-Q_{k}^{*}}\left|K(x-y)-K\left(x-y_{k}\right)\right||x|^{\alpha} d x\right) d y \\
<\sum \int_{Q_{k}}|b(y)|\left(\frac{c}{\left|Q_{k}\right|} \int_{Q_{k}}|x|^{\alpha} d x\right) d y \\
<c \sum \int_{Q_{k}}|b(y)||y|^{\alpha} d y<c \int_{\mathbf{R}^{n}} f(y)|y|^{\alpha} d y .
\end{aligned}
$$

Theorem 1 now follows as usual.

Proof of Theorem 2. Consider the case $n=2$. Define $\Omega(\theta)$ of period $2 \pi$ by

$$
\Omega(\theta)= \begin{cases}1 /|\theta|\left|\log ^{3}\right| \theta||, & |\theta|<1 / 2, \\ -1 /|\theta-\pi|\left|\log ^{3}\right| \theta-\pi \|, & |\theta-\pi|<1 / 2, \\ 0, & \text { elsewhere in }|\theta|<\pi .\end{cases}
$$

Clearly, $\Omega$ has mean-value zero. To see it satisfies (1), let $|\rho|<\delta, \delta<\frac{1}{8}$. By symmetry and the fact that $\Omega(\theta)=\Omega(\theta+\rho)=0$ if $\frac{1}{2}+\delta<|\theta|<\pi-\frac{1}{2}-\delta$,

$$
\begin{aligned}
\int_{-\pi}^{\pi}|\Omega(\theta+\rho)-\Omega(\theta)| d \theta & =2 \int_{|\theta|<1 / 2+\delta}|\Omega(\theta+\rho)-\Omega(\theta)| d \theta \\
& \leqslant 4 \int_{|\theta|<3 \delta}|\Omega(\theta)| d \theta+2 \int_{2 \delta<|\theta|<1 / 2+\delta}|\Omega(\theta+\rho)-\Omega(\theta)| d \theta \\
& =D_{1}+D_{2} .
\end{aligned}
$$

We have

$$
\begin{aligned}
& D_{1}=8 \int_{0}^{3 \delta} \frac{d \theta}{\theta\left|\log ^{3} \theta\right|}=\frac{c}{\log ^{2} 3 \delta}, \\
& D_{2} \leqslant 2 \int_{2 \delta<|\theta|<1 / 2-\delta}|\Omega(\theta+\rho)-\Omega(\theta)| d \theta+4 \int_{1 / 2-2 \delta<|\theta|<1 / 2+2 \delta}|\Omega(\theta)| d \theta .
\end{aligned}
$$

Since $\delta$ is small, $\Omega(\theta)$ is bounded for $\frac{1}{2}-2 \delta<|\theta|<\frac{1}{2}+2 \delta$. Thus the second term in the estimate for $D_{2}$ is bounded by $c \delta$. In the first term, $|\theta+\rho|$ is equivalent to $|\theta|$ since $|\theta|>2 \delta$ and $|\rho|<\delta$. Hence, by the mean-value theorem, the first term is at most $c \delta \int_{2 \delta<|\theta|<1 / 2-\delta} d \theta / \theta^{2}\left|\log ^{3}\right| \theta||<c \delta \int_{\delta}^{1 / 2} d \theta / \theta^{2}\left|\log ^{3} \theta\right|$. This, however, is bounded by $c(\log \delta)^{-3}$, as we now show. In fact, changing variables, we see the estimate in question reduces to $\int_{1}^{t}\left(e^{x} / x^{3}\right) d x<c e^{t} / t^{3}, t>1$. However,

$$
\int_{1}^{t} \frac{e^{x}}{x^{3}} d x \leqslant e^{t / 2} \int_{1}^{t / 2} \frac{d x}{x^{3}}+\frac{1}{(t / 2)^{3}} \int_{t / 2}^{t} e^{x} d x<c\left[e^{t / 2}+\frac{e^{t}-e^{t / 2}}{t^{3}}\right]<c \frac{e^{t}}{t^{3}} .
$$

Collecting estimates, we see that the $L^{1}$-modulus of continuity $\omega(\delta)$ of $\Omega$ satisfies $\omega(\delta)<c\left[\delta+(\log 3 \delta)^{-2}+|\log \delta|^{-3}\right]$ for small $\delta$, so that (1) holds. 
Now, let $K(x)=\Omega\left(x^{\prime}\right) /|x|^{2}$ be defined by (6) and let $f(x)=\chi(|x|<1)$, so that $f \in L_{|x|^{\alpha}}^{p}\left(\mathbf{R}^{2}\right)$ if $\alpha>-2$ and $1 \leqslant p<\infty$. Let $S$ be the strip $S=\left\{x=\left(x_{1}, x_{2}\right) \in \mathbf{R}^{2}\right.$ : $\left.x_{1} \geqslant 10,\left|x_{2}\right|<\frac{1}{2}\right\}$. If $x \in S$ and $|y|<1$, then $\Omega(x-y)>0$. Therefore, for $x \in S$,

$$
T f(x)=\int_{|y|<1} \frac{\Omega(x-y)}{|x-y|^{2}} d y \geqslant \frac{c}{|x|^{2}} \int_{|y|<1} \Omega(x-y) d y=\frac{c}{|x|^{2}} \int_{|x-y|<1} \Omega(y) d y .
$$

Hence, there is a constant $c_{1}$ so that

$$
T f(x) \geqslant \frac{c}{|x|^{2}} \int_{|x|-c_{1}}^{|x|+c_{1}} \rho d \rho \int_{|\theta|<c_{1} /|x|} \frac{d \theta}{\left.|\theta||\log | \theta\right|^{3}}, \quad x \in S .
$$

A simple computation gives $T f(x) \geqslant c /|x| \log ^{2}|x|, x \in S$. This implies

$$
\begin{aligned}
\int_{\mathbf{R}^{2}}|T f(x)|^{p}|x|^{\alpha} d x & \geqslant c \int_{S|x|^{p} \log ^{2 p}|x|} \\
& \geqslant c \int_{10}^{\infty} \rho^{\alpha-p+1}(\log \rho)^{-2 p}\left(\int_{0}^{c / \rho} d \theta\right) d \rho=c \int_{10}^{\infty} \rho^{\alpha-p}(\log \rho)^{-2 p} d \rho .
\end{aligned}
$$

The last integral is $+\infty$ if $\alpha>p-1$. This proves the part of Theorem 2 for $\alpha>p-1, p>1$. The part for $\alpha<-1, p>1$, follows by duality.

If $p=1$, the estimates above show that $\{x:|T f(x)|>\lambda\}$ contains the part of $S$ with $|x|<c_{\beta} \lambda^{-1 / \beta}$ for any $\beta>1$. It follows easily that the integral with respect to $|x|^{\alpha} d x$ over this set cannot be $O\left(\lambda^{-1}\right)$ for small $\lambda$ unless $\alpha<\beta-1$. This proves the part of Theorem 2 for $p=1, \alpha>0$. For $p=1$ and $\alpha<-1$, let $f_{N}$ be the characteristic function of the part of $S$ with $|x|<N, N$ large. Computations like those above show that $\int_{\mathbf{R}^{2}}\left|f_{N}(x)\right||x|^{\alpha} d x$ is bounded in $N$ if $\alpha<-1$, and that $T f_{N}(x) \geqslant c \log \log N$ for $x$ in a small neighborhood of 0 independent of $N$. If we choose $\lambda=c \log \log N$, it follows that (3) cannot hold for $w(x)=|x|^{\alpha}, \alpha<-1$.

The same technique can be used in $\mathbf{R}^{n}, n>2$, even for other kinds of Dini conditions. We say $\Omega$ satisfies the $L^{r}$-Dini condition, $1<r<\infty$, if (1) holds with $\omega$ defined in terms of the $L^{r}(|x|=1)$ norm. Fix $\xi$ in $\mathbf{R}^{n}$ with $|\xi|=1$, and for $|x|=1$, define $\Omega_{r}(x)=|x-\xi|^{-(n-1) / r}|\log | x-\left.\xi\right|^{-3}$ if $|x-\xi|<\frac{1}{2}, \quad \Omega_{r}(x)=$ $-|x+\xi|^{-(n-1) / r}|\log | x+\left.\xi\right|^{-3}$ if $|x+\xi|<\frac{1}{2}$, and $\Omega_{r}(x)=0$ otherwise. As before, $\Omega_{r}$ satisfies the $L^{r}$-Dini condition, but (2) fails for $f(x)=\chi(|x|<1)$ if $w(x)=|x|^{\alpha}$, $\alpha>p-1+(n-1) p / r^{\prime}$. If $p>1$, duality shows (2) also fails if $\alpha<-1-$ $(n-1) p / r^{\prime}$. As in [7], if $\Omega\left(x^{\prime}\right)$ is +1 for $x_{n}>0$ and -1 for $x_{n}<0, \Omega$ satisfies the $L^{r}$-Dini condition, $1 \leqslant r<\infty$, but (2) fails when $\alpha>n(p-1)$ or, by duality, $\alpha<-n$. These results thus agree with the negative conclusions of Theorem 1 of [7]. If $p=1$, analogous facts again hold for (3).

Proof of Theorem 3. Fix $s, l$ and $p$ with $1<s<2$, $[n / s]<l<n, l$ an integer, and $n / l<p \leqslant(n / l)^{\prime}$. Since $l<n$ and $p>1$, there exists $\beta>0$ with $l p-n<\beta$ $<n(p-1)$. Choose $\gamma<0$ so that $-n<\gamma<\beta-$ lp. (If $p=2$, we may take $l<\beta=-\gamma<n$.) Let $|\eta|=1$ and define $m(x)=e^{i x \cdot \eta}\left(1+|x|^{2}\right)^{-l / 2}, \quad w(x)=$ $|x|^{\beta}|x-\eta|^{\gamma}$. Then $m \in M(s, l)$, as was shown in [5], and $w \in A_{p}$, which follows either by direct computation or by appealing to the result of [4]. As in [5], if $\delta p>1$, the function

$$
f(x)=\left.|x|^{-(n+\beta) / p}|\log | x\right|^{-\delta} \chi(\{x:|x|<\mu\})
$$


is in $L_{w}^{p}$, and if $|x-\eta|<\mu / 2, T f(x) \geqslant c|x-\eta|^{l-(n+\beta) / p}|\log | x-\eta \|^{-\delta}(\mu>0$ depends on $l$ ). Thus, Tf $\notin L_{w}^{p}$ since $\{l-(n+\beta) / p\} p+\delta=l p-n-\beta+\gamma<$ $-n$.

\section{REFERENCES}

1. A. P. Calderon, M. Weiss and A. Zygmund, On the existence of singular integrals, Proc. Sympos. Pure Math., vol. 10, Amer. Math. Soc., Providence, R. I., 1967, pp. 56-73.

2. A. P. Calderón and A. Zygmund, On singular integrals, Amer. J. Math. 78 (1956), 289-309.

3. A A note on singular integrals, Studia Math. 65 (1979), 77-87.

4. P. W. Jones, Factorization of $A_{p}$ weights, Ann. of Math. (2) 111 (1980), 511-530.

5. D. S. Kurtz and R. L. Wheeden, Results on weighted norm inequalities for multipliers, Trans. Amer. Math. Soc. 255 (1979), 343-362.

6. B. Muckenhoupt, Weighted norm inequalities for the Hardy maximal function, Trans. Amer. Math. Soc. 165 (1972), 207-226.

7. B. Muckenhoupt and R. L. Wheeden, Weighted norm inequalities for singular and fractional integrals, Trans. Amer. Math. Soc. 161 (1971), 249-258.

8. E. M Stein, Singular integrals and differentiability properties of functions, Princeton Math. Ser., no. 30, Princeton Univ. Press, Princeton, N. J., 1970.

Department of Mathematics, Purdue University, West Layfayette, Indiana 47907

Department of Mathematics, Rutgers University, New Brunswick, New Jersey 08903 